\title{
Unusual venous thrombosis in a 35-year-old man
}

\section{Ami Schattner MD}

$\mathrm{A}$ n otherwise healthy 35-year-old man presented to the emergency department with substantial pain in his upper abdomen that had lasted for several days and was radiating to his back. The patient had experienced a similar episode 4 months earlier that had not been investigated.

On physical examination, the patient had hypertension (150/105 $\mathrm{mm} \mathrm{Hg})$, hepatosplenomegaly and pitting leg edema, with no signs of chronic liver disease. The results of laboratory investigations showed a hemoglobin level of 95 (normal 120-150) g/L, a leukocyte count of 9.5 (normal $4.0-10.0) \times 10^{\circ} / \mathrm{L}$ and a platelet count of 524 (normal $150-400) \times 10^{9} / \mathrm{L}$. The patient's erythrocyte sedimentation rate and C-reactive protein and ferritin levels were normal. Serum creatinine was elevated (159.1 [normal 62133] $\mu \mathrm{mol} / \mathrm{L})$, with proteinuria $(0.64 \mathrm{~g} / 24 \mathrm{~h})$ and normal sediment. The international normalized ratio was elevated (1.25 [normal 1.0]), as were levels of bilirubin (8.55 [normal $0-5.1] \mu \mathrm{mol} / \mathrm{L}$ ) and $\gamma$-glutamyl transferase (491.9 [normal 10.849.8] U/L). Serum alkaline phosphatase, albumin and aminotransferases were normal.

Duplex ultrasonography of the patient's abdomen showed an extensive, nearly occlusive thrombus in the infra- and intrahepatic inferior vena cava. The hepatic vein did not appear to be occluded, but the patchy appearance of the liver parenchyma and flow through a collateral vessel were consistent with obstruction of the hepatic outflow tract (Figure 1). Contrast-enhanced computer tomography $(\mathrm{CT})$ and magnetic resonance imaging (MRI) of the abdomen and chest confirmed hepatomegaly $(20 \mathrm{~cm}$, with enlargement of the caudate lobe), splenomegaly $(20 \mathrm{~cm})$, thrombosis of the inferior vena cava extending to below the renal veins and mild ascites (Figure 2). We saw no congenital membranous web, anomaly or compression of the inferior vena cava. Results of echocardiography (including the proximal inferior vena cava) were normal, and endoscopy showed no varices.

We started low-molecular-weight heparin ther- apy for thrombosis and ramipril for hypertension. The results of tests for heritable and acquired thrombophilia were negative. ${ }^{1}$ However, a review of the patient's earlier primary care charts showed thrombocytosis $\left(531-741 \times 10^{9} / \mathrm{L}\right)$ on 5 routine blood tests over the previous 6 years. The patient's family history was noncontributory.

The combination of thrombocytosis, which did not appear to be reactive or cytokine-driven (Box 1), ${ }^{2}$ and substantial splenomegaly, despite only mild portal hypertension and thrombosis, suggested myeloproliferative neoplasm. On reviewing the smear of the patient's peripheral blood, we saw large abnormal platelets. Bone marrow aspiration and biopsy showed a hyperplastic marrow with megakaryocyte proliferation, atypia and clustering, increased eosinophils and increased reticulin fibres (grade 1/3). Genetic testing showed the presence of the JAK2 V617F mutation, with low (20\%) JAK2 mutant allele burden; the results of testing for the $B C R-$ $A B L$ fusion gene were negative. We diagnosed essential thrombocythemia and started the patient on hydroxyurea $(1000 \mathrm{mg} / \mathrm{d})$ and warfarin. After 1 year of follow-up, the patient feels well and remains symptom-free.

\section{Discussion}

The patient's pain, edema, cholestatic liver enzymes, renovascular hypertension and protein-

\section{KEY POINTS}

- Patients presenting with venous thromboembolism may undergo testing indiscriminately for heritable and acquired thrombophilia, but this practice is unsupported by guidelines.

- Traditional clinical methods remain important in evaluating venous thromboembolism; selective cancer screening may be warranted in patients more than 40 years of age with an unprovoked event.

- Malignant disease, local factors, cirrhosis of the liver and myeloproliferative neoplasms are among the most common risk factors for abdominal venous thrombosis.

- Testing for the JAK2 mutation is strongly indicated for patients with splanchnic vein thrombosis, and a positive result may suggest polycythemia vera or essential thrombocythemia. 
uria on presentation were due to an extensive thrombosis of the inferior vena cava, an uncommon occurrence. In a large registry of venous thromboembolism, 53 of 1770 patients $(3.0 \%)$ had thrombosis of the inferior vena cava. ${ }^{3}$ Thrombosis of the inferior vena cava can be caused by an adjacent tumour (e.g., renal, adrenal, hepatic, pancreatic, retroperitoneal) or local pathology (e.g., congenital membranous web, endovascular devices, external compression, severe infection in an area drained by the vessel). ${ }^{3}$ These causes were excluded in our patient, but several factors (organ enlargement, abnormal platelet count and idiopathic intraabdominal venous thrombosis) suggested an underlying disease and mandated a careful search for a thrombophilic disorder, after ruling out reactive thrombocytosis (Box 1$)^{2}$

\section{Establishing the cause of venous thromboembolism}

Once clinically suspected venous thromboembolism is confirmed by imaging, establishing the cause comes second only to treatment. First-time venous thromboembolism occurs in about 100 people per 100000 each year in the United States, with the rate rising exponentially with age. ${ }^{1}$ The proportion of idiopathic cases of a first venous thromboembolism (i.e., not associated with known cancer or temporary precipitating circumstances such as antecedent trauma, surgery, immobilization, pregnancy, puerperium or estrogen use) is as high as $55 \%$. $^{4}$ Thus, many

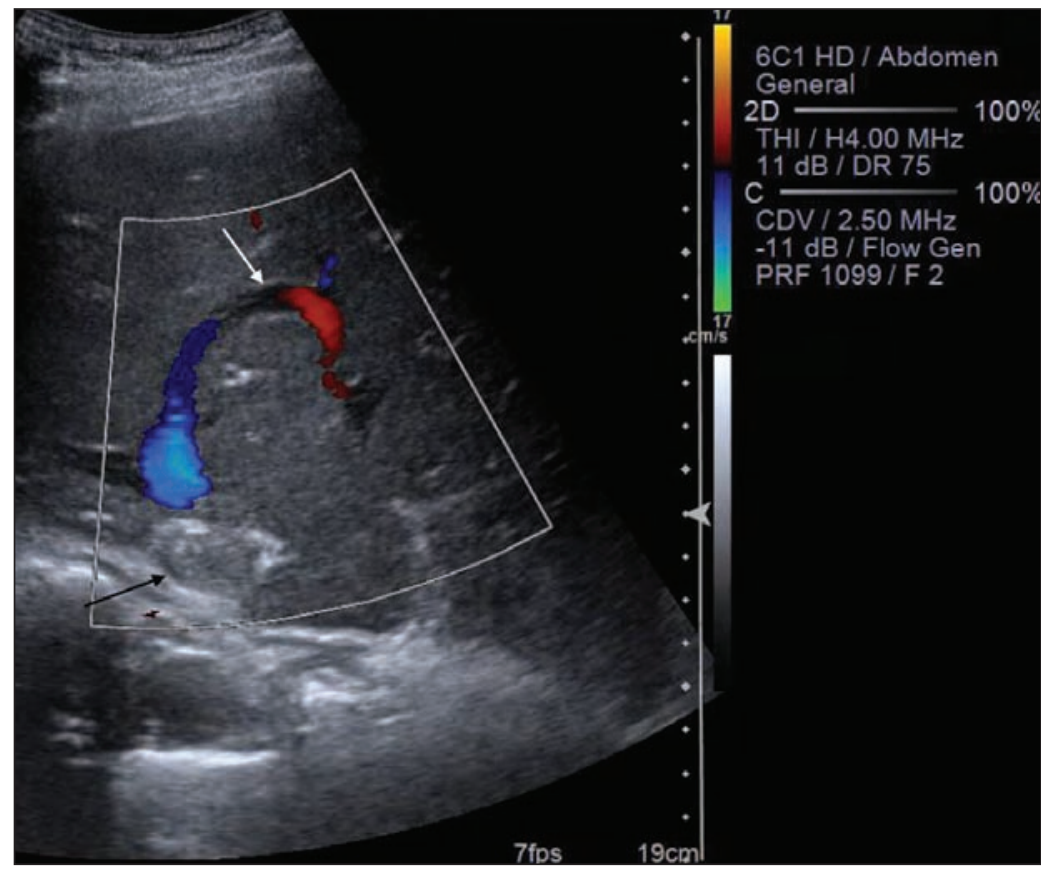

Figure 1: Colour doppler sonograph of the liver of a 35-year-old man, showing a thrombosed inferior vena cava (black arrow) and a typical curved venovenous intrahepatic collateral vessel bypassing the occlusion (white arrow). patients are potential candidates for a wide array of tests (Box 2), ${ }^{1,4-16}$ and the rapid increase in the understanding of thrombophilic mechanisms has led to a growing pressure for indiscriminate testing. However, this approach is not supported by the evidence. Although prospective studies analyzing a comprehensive screening protocol and its impact in consecutive cases of venous thromboembolism are not available, several suggestions around screening can be made.

A traditional clinically based approach remains best practice. ${ }^{5}$ A thorough history, examination and basic laboratory investigations will identify patients with precipitating circumstances, local factors (e.g., central venous catheter or pacemaker, varicose veins), active malignant disease or a systemic disease associated with hypercoagulability (such as systemic lupus erythematosus, inflammatory bowel disease, nephrotic syndrome, chronic kidney disease, cirrhosis of the liver or HIV infection). ${ }^{5}$ These patients are considered to have a provoked venous thromboembolism and (with the exception of antiphospholipid syndrome in patients with lupus) need no further testing, ${ }^{6,7}$ even though they may also have a concurrent thrombophilia. ${ }^{1}$

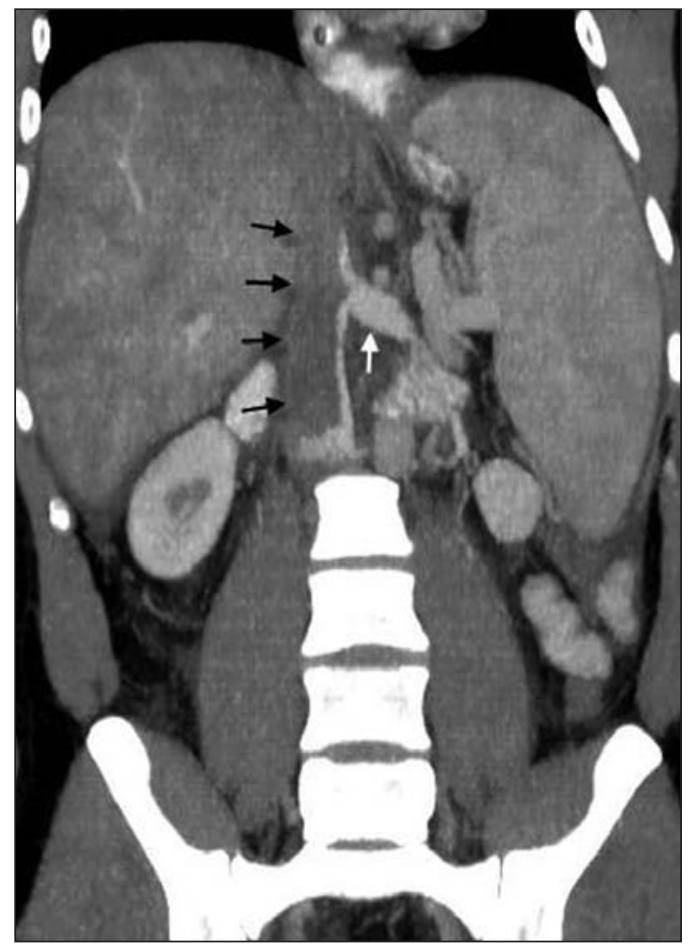

Figure 2: Contrast-enhanced computed tomography image of the abdomen (coronal view, maximum intensity projection and multiplanar reformation) showing extensive thrombosis of the inferior vena cava below the liver (black arrows), with dilation of the left renal vein (white arrow) and hepatosplenomegaly. Heterogeneity of the parenchyma of the liver can be seen, consistent with obstruction of hepatic outflow. 
A substantial proportion of patients with an unprovoked thromboembolism (about 1 in 10) have cancer. ${ }^{8}$ Prospective observational studies have shown that almost one-half of these cancers are occult and will not be detected by either routine or limited evaluation. ${ }^{8}$ Many more cancers (but not all) may be detected by more extensive screening, particularly CT imaging of the abdomen and pelvis with or without chest imaging or testing for tumour markers. For example, occult cancers were found in $13 \%$ of patients with acute idiopathic venous thromboembolism who who were randomly assigned to an extensive screening protocol, and this group had earlier stage cancer and lower mortality compared with patients in the control group who did not undergo screening and whose cancers were found incidentally. ${ }^{8}$ Despite supportive data from a good-quality systematic review of 36 studies, ${ }^{9}$ neither cost-effectiveness nor survival benefit of screening for occult cancer in unprovoked venous thromboembolism has hitherto been proven. Nevertheless, recent guidelines endorse CT imaging in patients over 40 years of age with an unprovoked thromboembolism, ${ }^{7}$ a diagnosis that has clear implications both for the choice of treatment (e.g., low-molecular-weight heparin v. warfarin) and its duration.

A battery of screening tests for hereditary thrombophilia is currently available (Box 2), but indiscriminate, unselected screening for patients and their relatives is not supported by evidence from prospective studies and is not recommended by guidelines. ${ }^{67,10}$ The practice is expensive and not cost-effective, a potential cause of undue anxiety, often affected by problems of standardization and, most of all, not proven to have treatment implications or affect the probability of the condition's recurrence. ${ }^{6,11}$ The most common hereditary thrombophilia is heterozygous FVL (about 21\%) or a prothrombin mutation (about $8 \%$ ). ${ }^{1}$ However, the increase in rate of recurrence among carriers of these common mutations is not substantial. One-third of patients with recurrent unprovoked venous thromboembolism have normal test results $;{ }^{12}$ finding 1 or more thrombophilic factors in the remainder is likely, but will not typically predict recurrence nor alter the clinical management of the condition in most patients., ${ }^{4,13}$ Moreover, in a study involving 474 patients with a first thrombotic event followed for a mean of 7 years, recurrent events were not only equal in those with and without inherited prothrombotic abnormality (excluding antiphospholipid syndrome, which was not studied), but were uncommon (33.6 per 1000 patient-years). ${ }^{4}$ In contrast, warfarin prophylaxis continued indefinitely is associated with a substantial cumulative risk of major bleeding (typically, 1\%-3\% per person-year), which may outweigh any potential benefit of treatment. ${ }^{10,11,13}$

Selective screening can be useful despite the lack of solid data supporting it. Sometimes, screening may be motivated by patients' wishes. Moreover, the cumulative chance of identifying a strong thrombophilic risk factor (e.g., antiphospholipid syndrome, natural anticoagulant deficiency, homozygous or combined mutations) after an unprovoked thromboembolism is substantial, possibly around $15 \%$ in white populations..$^{1,4,13,14}$ Thus, a clinically based decision to test for these factors may be sound in a patient with a first unprovoked venous thromboembolism appearing at a younger age (e.g., less than 50 years) or in the presence of a highly positive family history of early idiopathic thrombosis or recurrent fetal loss in first-degree relatives. ${ }^{14}$ Finding a strong thrombophilic risk factor after an unprovoked thromboembolism with no evidence of cancer may support a recommendation for extended (potentially lifelong) treatment with warfarin or the newer anticoagulants in patients deemed at low risk of bleeding. Recent guidelines stress that even unselected patients with unprovoked venous thromboembolism have an annual recurrence rate higher than $5 \%(10 \%$ for the first year) and should be considered for long-term anticoagulant therapy provided their bleeding risk estimates are low and the patients agree. ${ }^{15}$ Even for patients whose bleeding risk may preclude extended

Box 1: Conditions that may cause elevated ( $\left.>400 \times 10^{3} / \mu \mathrm{L}\right)$ thrombocyte counts in peripheral blood*2

\section{Reactive (cytokine-driven)t}

- Infectiont (e.g., community-acquired pneumonia, tuberculosis, infective endocarditis, abscess, soft-tissue infection)

- Chronic inflammatory disease (e.g., systemic vasculitis, inflammatory bowel disease, rheumatoid arthritis)

- Paraneoplastic syndrome (e.g., lung cancer, ovarian cancer, gastrointestinal cancer, hypernephroma, lymphoma)

- Splenectomy or functional hyposplenism (associated with many immunemediated diseases [e.g., celiac disease], as well as hematological diseases and portal hypertension; may be overlooked unless the peripheral blood smear is carefully examined)

- Iron-deficiency anemia

- Acute hemorrhage or acute hemolysis

- Withdrawal of ethanol, methotrexate or chemotherapy causing thrombocytopenia; treatment of $B_{12}$ deficiency or idiopathic thrombocytopenic purpura; or drug-induced by vincristine

\section{Clonal (autonomous)}

- Myeloproliferative neoplasm (essential thrombocythemia, polycythemia vera, primary myelofibrosis, chronic myeloid leukemia)

- Myelodysplastic disorder (especially associated with chromosome 5q deletion syndrome, refractory anemia with ringed sideroblasts with thrombocytosis and myelodysplastic/myeloproliferative neoplasms)

*Counts in the range of $400-500 \times 10^{3} / \mu \mathrm{L}$ may occur normally in about $1 \%$ of the general population and are often transient.

tReactive thrombocytosis is the predominant cause of thrombocytosis $(70 \%-89 \%)$, and infection is its most common cause $(31 \%-48 \%)$ in sequential patient series reported. 
therapy, heightened awareness may contribute to secondary prevention in high-risk circumstances. In patients with definite antiphospholipid syndrome and thrombosis, anticoagulation for an indefinite period may be warranted. ${ }^{13}$ In addition, intra-abdominal thrombosis in the absence of underlying disease is strongly associated with myeloproliferative neoplasms, paroxysmal nocturnal hemoglobinuria or antiphospholipid syndrome ${ }^{3}$ and, as in our patient's case, guidelines recommend screening for these conditions. ${ }^{16}$

Box 2: Potential investigations for thrombophilic risk factors in patients with unprovoked first venous thrombosis*1,4-16

\section{Inherited risk factors $†$}

Factor V Leiden (predominantly in white people)

- Partial thromboplastin time-based assay for activated protein C resistance, confirmed by genomic DNA analysis for factor $V$ mutation after polymerase chain reaction amplification of mononuclear cells.

Prothrombin (factor II) gene mutation

- Detection of G20210A mutation by PCR amplification of DNA from whole blood.

- Primary protein C, protein S or antithrombin deficiency Low level seen (not to be tested during acute venous thromboembolism or anticoagulation) and confirmed in a first-degree relative.

High levels of factor VIII

- More than $1500 \mathrm{IU} / \mathrm{mL}$ ( $150 \%$ of normal).

\section{Acquired risk factors}

Pregnancy, postpartum or exogenous female sex hormones

- Taking patient's history.

Occult cancer

- Clinical clues should be followed. When negative, consider screening with an abdominopelvic computed tomography scan in all patients more than 40 years of age, with or without chest imaging or testing for tumour markers.

Myeloproliferative malignant disease

- JAK2 mutation in peripheral granulocyte DNA.

Paroxsymal nocturnal hemoglobinuria

- Flow cytometry of erythrocytes (or granulocytes) using monoclonal antiCD55 and anti-CD59, showing an absence of glycophosphatidylinositol-anchored proteins. Fluorescent aerolysin cytometry is more sensitive and specific.

Antiphospholipid syndrome

- Lupus anticoagulant (strongest association with thrombosis; requires testing before starting treatment with an anticoagulant) and antibodies against cardiolipin and $\beta_{2}$ glycoprotein; any positive results should be confirmed at least 12 weeks later.

Behcet disease

- History and examination using International Study Group criteria.

Celiac disease

- Immunoglobulin A antiendomysial antibodies or anti-tissue transglutaminase antibodies.

Hyperhomocysteinemia (genetic MTHFR mutation less common than deficiency of folic acid, vitamin $\mathrm{B}_{6}$ or $\mathrm{B}_{12}$ )

- Fasting serum homocysteine level higher than $18.5 \mu \mathrm{mol} / \mathrm{L}$.

*Not routinely recommended in unselected patients (see text).

+Venous thromboembolism is a chronic disease with multicausal pathogenesis and a high rate of recurrence. Often, a combination of genetic (multigenic) and acquired risk factors coexist. In contrast with many acquired risk factors, an association of hereditary thrombophilia with arterial thrombosis is generally absent.

\section{Essential thrombocythemia}

Our patient fulfilled established World Health Organization (WHO) criteria for essential thrombocythemia, ${ }^{17}$ with reactive (or cytokine-driven) thrombocytosis ruled out (Box 1). In a case series involving 53 patients with inferior vena cava thrombosis, thrombophilia was the most common risk factor, found in more than half of the patients; overt myeloproliferative neoplasm was found in up to half of the patients with hepatic vein thrombosis and up to one-third of those with extrahepatic portal vein thrombosis. ${ }^{3}$

Essential thrombocythemia mostly affects older adults, but about $20 \%$ of cases occur in young adults, such as our patient. ${ }^{18}$ More than one-third of patients have vasomotor symptoms (e.g., headache, lightheadedness, syncope, atypical chest pain, acral paresthesia or erythromelalgia). ${ }^{18}$ As in our patient's case, thrombosis may be the presenting manifestation $(12 \%-39 \%$ of patients with polycythemia vera; $11 \%-25 \%$ of patients with essential thrombocythemia). ${ }^{19}$ Because other features of myeloproliferative neoplasms may not be evident, guidelines strongly recommend that JAK2 screening be included in the workup of thrombophilia. ${ }^{16}$ In patients with essential thrombocythemia who do not have the V617F mutation $(<50 \%)$, the condition is diagnosed by bone marrow biopsy, and these patients appear to have fewer venous thrombotic events.

Treatment decisions in essential thrombocythemia are guided by risk stratification (riskadapted therapy), which targets primarily thrombotic complications, based on an international cohort of 891 patients with essential thrombocythemia defined by WHO criteria. ${ }^{18}$ For high-risk patients, the only randomized study comparing drug to no drug, found $3.6 \%$ thrombotic episodes in patients treated with hydroxyurea versus $24 \%$ without such treatment. ${ }^{17}$ These are important findings because thrombotic complications (arterial or venous) constitute the major cause of morbidity and mortality in essential thrombocythemia, although hemorrhage, thrombosis or evolution to myelofibrosis or leukemia affects just $1.29 \%$ per year of younger patients with essential thrombocythemia, ${ }^{18}$ and most patients can expect nearnormal survival.

Thrombotic events in essential thrombocythemia are likely to be multifactorial in their pathogenesis. ${ }^{19}$ However, increasing clinical and laboratory evidence supports a role for the $J A K 2$ mutation in thrombosis, as seen in our patient's case. His positive test result could suggest either a heterozygous or a homozygous mutation present in a small clone. In this context, the evolving therapeutic potential of JAK2 inhibitors is an intriguing development. 


\section{References}

1. Rosendaal FR. Venous thrombosis: a multicausal disease. Lance 1999;353:1167-73.

2. Rose SR, Petersen NJ, Gardner TJ, et al. Etiology of thrombocytosis in a general medicine population: analysis of 801 cases with emphasis on infectious causes. J Clin Med Res 2012;4:415-23.

3. Linnemann B, Schmidt H, Schinderwolf M, et al. Etiology and VTE risk factor distribution in patients with inferior vena cava thrombosis. Thromb Res 2008;123:72-8.

4. Christiansen SC, Cannegieter SC, Koster T, et al. Thrombophilia, clinical factors, and recurrent thrombotic events. JAMA 2005;293:2352-61.

5. Paley L, Zornitzki T, Cohen J, et al. Utility of clinical examination in the diagnosis of emergency patients admitted to the department of medicine of an academic hospital. Arch Intern Med 2011;171:1394-6.

6. Baglin T, Gray E, Greaves M, et al. Clinical guidelines for testing for heritable thrombophilia. Br J Haematol 2010;149:209-20.

7. Venous thromboembolic diseases: the management of venous thromboembolic diseases and the role of thrombophilia testing. London (UK): National Institute for Health and Clinical Excellence; 2012.

8. Piccioli A, Lensing AW, Prins $\mathrm{MH}$, et al. Extensive screening for occult malignant disease in idiopathic venous thromboembolism: a prospective randomized clinical trial. J Thromb Haemost 2004;2:884-9.

9. Carrier M, Le Gal G, Wells PS, et al. Systematic review: the Trousseau syndrome revisited: Should we screen extensively for cancer in patients with venous thromboembolism? Ann Intern Med 2008;149:323-33.

10. Kearon C, Akl EA, Comerota AJ, et al. Antithrombotic therapy for VTE disease. Antithrombotic therapy and prevention of thrombosis, 9th ed: American College of Chest Physicians Evidence-based clinical practice guidelines. Chest 2012;141(suppl 2):e419Se494S.

11. Segal JB, Brotman DJ, Necochea AJ, et al. Predictive value of Factor V Leiden and prothrombin G20210A in adults with venous thromboembolism and in family members of those with a mutation: a systematic review. JAMA 2009;301:2472-85.

12. Kyrle PA, Rosendaal FR, Echinger S. Risk assessment for recurrent venous thromboembolism. Lancet 2010;376:2032-9.

13. Middeldorp S, van Hylckama Vlieg A. Does thrombophilia screening help in the clinical management of patients? Br J Haematol 2008; $143: 321-5$

14. Lijfering WM, Brouwer JL, Veeger NJ, et al. Selective testing for thrombophilia in patients with first venous thrombosis: results from a retrospective family cohort study on absolute thrombotic risk for currently known thrombophilic defects in 2479 relatives. Blood 2009;113:5314-22.

15. Baglin T, Bauer K, Douketis J, et al. Duration of anticoagulant therapy after a first episode of an unprovoked pulmonary embolus or deep vein thrombosis: guidance from the SSC of the ISTH. J Thromb Haemost 2012;10:698-702.

16. Tait $\mathrm{C}$, Baglin $\mathrm{T}$, Watson $\mathrm{H}$, et al. Guidelines on the investigation and management of venous thrombosis at unusual sites. $\mathrm{Br}$ Haematol 2012;159:28-38.

17. Tefferi A. Polycythemia vera and essential thrombocythemia: 2012 update on diagnosis, risk stratification, and management Am J Hematol 2012;87:285-93.

18. Barbui T, Thiele J, Carobbio A, et al. Disease characteristics and clinical outcome in young adults with essential thrombocythemia versus early/prefibrotic primary myelofibrosis. Blood 2012;120: 569-71.

19. Austin SK, Lambert JR. The JAK2 V617F mutation and thrombosis. Br J Haematol 2008;143:307-20.

Affiliations: Department of Medicine, Kaplan Medical Centre, Rehovot; Faculty of Medicine, Hebrew University Hadassah Medical School, Jerusalem, Israel
The section Cases presents brief case reports that convey clear, practical lessons. Preference is given to common presentations of important rare conditions, and important unusual presentations of common problems. Articles start with a case presentation (500 words maximum), and a discussion of the underlying condition follows (1000 words maximum). Visual elements (e.g., tables of the differential diagnosis, clinical features or diagnostic approach) are encouraged. Written consent from patients for publication of their story is a necessity and should accompany submissions. See information for authors at www.cmaj.ca. 